\title{
CONDUCTA PROSOCIAL \\ DE LOS ESTUDIANTES HACIA \\ LA DISCAPACIDAD Y RELACIÓN \\ CON LA INTELIGENCIA EMOCIONAL
}

\section{Prosocial conduct of students to disability and relationship with emotional intelligence}

\author{
Raquel Suriá MarTínez \\ Universidad de Alicante. Departamento de Comunicación y Psicología Social. Edificio de Ciencias \\ Sociales \\ raquel.suria@ua.es
}

Recepción: 6 de enero de 2018

Aceptación definitiva: 29 de agosto de 2018

Resumen: Este estudio identifica la relación entre IE y la conducta prosocial de los estudiantes hacia sus compañeros con discapacidad. Participaron 327 estudiantes universitarios a los que se les administró la Trait Meta-Mood Scale-24 (TMMS-24) y la Prosocialness Scale for Adults [PSA]. El análisis de conglomerados identificó tres perfiles diferenciados de IE. Asimismo, los grupos con alta IE y alta puntuación en comprensión y regulación obtuvieron mayores puntuaciones en conducta prosocial hacia la discapacidad. Estos resultados sugieren la necesidad de profundizar en la relación de IE y la conducta prosocial para diseñar programas que fomenten en los estudiantes conductas prosociales hacia la discapacidad.

Palabras Clave: conducta prosocial; estudiantes universitarios; discapacidad.

Aвstract: This study identifies the relationship between EI and prosocial behavior of students toward their peers with disabilities. Trait Meta-Mood Scale-24 (TMMS-24) and Prosocialness Scale for Adults [PSA] were administered to 327 university students. Cluster analyses allowed identifying four different EI profiles. Also, the Groups with high general EI and high scores in emotional regulation obtained higher scores in prosocial 
behavior domains than the other groups. Results suggest the need of going in depth on EI knowledge and the relationship with prosocial behavior to design programs that foster prosocial behaviors toward students with disabilities.

KEY WORDS: prosocial behavior; university students; disability.

\section{Introducción}

$\tau$

A EDUCACión INCLUSIVA es, sin duda, uno de los temas más importantes en el contexto educativo. Temas como la integración o la atención al alumnado con discapacidad llevan a que el sistema educativo desarrolle estrategias adecuadas para el desarrollo integral de los estudiantes con discapacidad (Ainscow, 2005; Arnáiz-Sánchez, 2012; Damm-Muñoz, 2014; Echeita y Simón, 2014; Suriá, 2016).

Para ello, una de las variables fundamentales son las actitudes y comportamientos del resto de compañeros hacia los estudiantes con discapacidad (De Boer, Pijl y Minnaert, 2010; Novo-Corti, Muñoz-Cantero y Calvo-Porral, 2011; Suriá, 2014), cobrando gran relevancia las conductas prosociales, especialmente las dirigidas a este colectivo (Inglés, Martínez-González y García-Fernández, 2015; Parra y Luque-Rojas, 2011; Redondo-Pacheco y Guevara-Melo, 2012).

De forma general, la conducta prosocial se define como la conducta de carácter voluntario y que beneficia a los demás, considerándose sinónimo de socialización (Spinrad et al., 2006; Martorell, González, Aloy y Ferris, 1995). En referencia a su definición, y aunque no existe unanimidad, la mayoría de autores incluyen el concepto “conducta social positiva” (Spinrad et al., 2006; Inglés et al., 2015). Así, forma parte de la personalidad, comprendiendo acciones de ayuda, cooperación e intercambio y altruismo en las relaciones afectivas (Brown y Ferris, 2004; Fehr y Fischbacher, 2003), en el cumplimiento de las normas sociales (Caprara y Pastorelli, 1993; Lim, Khoo y Wong, 2007) y fundamental en el funcionamiento psicológico y social del individuo (Hardy, Carlo y Roesch, 2010).

Este constructo ha sido estudiado desde distintas teorías, estableciéndose en todas ellas que sus diferentes componentes se relacionan con el desarrollo emocional y cognitivo de la persona, adquiriéndose a lo largo de diferentes etapas del ciclo vital, especialmente en edades tempranas como la adolescencia y la juventud, donde se incrementa la identificación y la conformidad con el grupo de iguales (Caprara, Alessandri y Eisenberg, 2012; Inglés et al., 2015; Olivar y Soza, 2014).

Con respecto a esto, diferentes estudios se han dedicado a la conducta prosocial y su asociación con diferentes variables sociodemográficas como el sexo (RedondoPacheco y Guevara-Melo, 2012) o la edad (Carlo, Crockett, Randall y Roesch, 2007).

Asimismo, la literatura existente ha abordado este constructo en los diferentes contextos educativos, es decir, en la etapa escolar (Del Barco, Castaño y Carroza, 2010), la etapa de enseñanza secundaria (Lozano y Etxebarria, 2007), así como en el ámbito universitario (Rivas-Damián, 2012), sin embargo, todos ellos están enfocados a las competencias sociales y conductas prosociales hacia la diversidad en general, 
desconociendo, por tanto, trabajos dedicados específicamente a la conducta prosocial hacia la discapacidad. En este sentido, al profundizar en la discapacidad, no se debe entender este concepto como algo global, sino que existen diferentes tipologías con características propias de cada tipo de discapacidad (p. e., discapacidad sensorial, intelectual, etc.), que pueden ser relevantes o más significativas a la hora de estudiar los comportamientos prosociales hacia las personas con alguna de estas discapacidades.

Además de las variables propias de la discapacidad, existen multitud de variables personales que pueden influir en estas conductas prosociales. Así, una de las variables poco estudiadas pero que está cobrando especial interés en el estudio de las competencias actitudinales y sociales es la inteligencia emocional (IE) (Gilar, Miñano y Castejón, 2008; Jiménez y López-Zafra, 2011; Morales y López-Zafra, 2013, entre otros).

Este concepto fue definido hace varias décadas por Mayer y Salovey (1997) como un constructo compuesto por diferentes habilidades encargadas de percibir, evaluar y expresar las emociones de forma adecuada, el acceso y generación de sentimientos facilitadores del pensamiento, la comprensión y, por último, del procesamiento y manejo de las emociones (Mayer y Salovey, 1997) y, por tanto, de la adaptación efectiva de las personas a su entorno (Morales y López-Zafra, 2013).

Basándose en esta definición, y aunque existen diferentes instrumentos para medir la IE, uno de los más utilizados es el Trait Meta-Mood Scale (TMMS), cuestionario elaborado por Salovey, Mayer, Goldman, Turvey y Palfai (1995) que identifica empíricamente tres dimensiones o habilidades involucradas en el razonamiento emocional: a) Percepción de las emociones, b) Comprensión emocional y c) Reparación de las emociones. Estos tres componentes se dirigen a la capacidad para identificar y reflexionar sobre las emociones (Percepción), a la comprensión de los propios estados emocionales (Comprensión) y, por último, a la regulación de los sentimientos (Reparación), evidenciándose que una elevada IE está asociada positivamente con el buen funcionamiento del individuo en diferentes ámbitos (Brackett, Rivers, Shiffman, Lerner y Salovey, 2006; Buenrostro et al., 2012; Martins, Ramalho y Morin, 2010), así como con unas adecuadas habilidades y competencias sociales interpersonales.

De esta forma, las personas con niveles elevados de IE promueven el razonamiento flexible, la capacidad de extender los repertorios de acción e integración, la preferencia hacia lo variado en lugar de lo rígido (Mayer y Salovey, 1997; Morales y LópezZafra, 2013) y, con ello, favorecen en la persona la tendencia a comprender y ayudar al otro a través de conductas solidarias, lo cual implicaría una mejor integración social (Buenrostro et al., 2012; Salovey et al., 1995).

Si se echa una mirada a la taxonomía de IE propuesta por Lazarus (2000) se incluye un tipo particular de emociones positivas: las empáticas. Estas surgen en el marco de la interacción social y requieren la capacidad de empatizar con los otros, lo cual implica sensibilidad para con las emociones y necesidades de otras personas y, por tanto, ser un poderoso motivador de la conducta prosocial (Bandura, Caprara, Barbaranelli, Gerbino y Pastorelli, 2003; Caprara, Alessandri y Eisenberg, 2012; Hardy et al., 2010; Inglés et al., 2015).

Si se echa una mirada a la literatura publicada sobre la IE y su relación con la conducta prosocial de los estudiantes hacia sus compañeros con discapacidad, en 
la actualidad no se han encontrado trabajos previos que analicen en profundidad esta relación. Centrándonos en la IE y en los componentes que la conforman, son escasos los estudios enfocados a analizar la existencia de combinaciones de los componentes de IE (Atención, Comprensión y Regulación emocional) que den lugar a diferentes perfiles de este constructo en función de la relevancia de cada componente entre los estudiantes universitarios.

A su vez, son inexistentes los estudios publicados sobre diferencias en conducta prosocial entre distintos perfiles de IE de los estudiantes que tengan en cuenta no solo la conducta prosocial general, sino también otras dimensiones que conforman este constructo (p. e., altruismo, compartir, cuidar y empatía). La existencia de diferentes perfiles podría esclarecer qué perfil es más idóneo para una mayor conducta prosocial entre los estudiantes y, por tanto, para favorecer la integración de los estudiantes con discapacidad.

A partir de estas consideraciones, en el presente estudio se plantean tres objetivos. En primer lugar, identificar si entre los participantes existen combinaciones de diferentes dimensiones de IE que den lugar a diferentes perfiles, los cuales podrían definirse en función del peso que tenga cada una de las dimensiones dentro de cada perfil.

H1. Existen perfiles de IE diferentes en función del peso que cobre cada componente de IE.

En segundo lugar, una vez hallados y definidos los perfiles de IE, se analiza si existen diferencias estadísticamente significativas entre los perfiles definidos y las diferentes puntuaciones en conducta prosocial. Este objetivo del estudio constituye, en cierto sentido, la validación criterial de los perfiles encontrados y su utilidad a la hora de diseñar programas de entrenamiento que potencien la IE y la conducta prosocial de los estudiantes universitarios y, con ello, que fomenten de forma activa la integración de los estudiantes con discapacidad. De este objetivo se desprende que:

H2. Existen diferencias estadísticamente en la conducta prosocial en función de los perfiles de IE.

Finalmente, el tercer objetivo explora si la conducta prosocial de los estudiantes hacia las personas con discapacidad puede estar influida por la tipología de la discapacidad, en concreto, se espera que:

H3. Existan diferencias estadísticamente significativas en la conducta prosocial en función del tipo de discapacidad.

\section{Método}

\subsection{Participantes}

Se ha estudiado la serie de casos transversal constituida por una muestra intencional -por motivos de accesibilidad-, de la Universidad de Alicante. En este caso participaron 327 estudiantes, con edades comprendidas entre los 18 y 30 años, siendo la 
media de edad de 21,52 (DT = 4,80). De ellos, $228(69,7 \%)$ eran mujeres y $99(30,3 \%)$ varones. Los alumnos procedían de distintas titulaciones (39,4\% de Magisterio, $20,8 \%$ de Relaciones Laborales, $18 \%$ de Criminología, 14,4\% de Psicopedagogía y $7,3 \%$ de Trabajo Social). La Tabla 1 presenta la distribución de la muestra por edad y sexo. A través de la prueba $\chi^{2}$ de homogeneidad de la distribución de frecuencias, se comprobó la ausencia de diferencias estadísticamente significativas entre los diez grupos de Sexo x Curso $\left(\chi^{2}=3,68 ; p=0,66\right)$.

\begin{tabular}{|l|r|c|}
\hline \multicolumn{3}{|c|}{ Tabla 1. Perfil sociodemográfico } \\
\hline Curso & \multicolumn{1}{c|}{ N } & \multicolumn{1}{c|}{$\%$} \\
\hline 2014 & 140 & 42,8 \\
\hline 2015 & 187 & 57,2 \\
\hline Mujer & 228 & 69,7 \\
\hline Hombre & 99 & 30,3 \\
\hline Magisterio & 129 & 39,4 \\
\hline RR. LL. & 68 & 20,8 \\
\hline Criminología & 59 & 18 \\
\hline Psicopedagogía & 47 & 14,4 \\
\hline Trabajo social & 24 & 7,3 \\
\hline 18-22 & 139 & 42,9 \\
\hline $23-26$ & 91 & 27,8 \\
\hline $27-30$ & 97 & 29,8 \\
\hline & 327 & 100 \\
\hline
\end{tabular}

\subsection{Instrumentos}

- Cuestionario sociodemográfico; se utilizó un cuestionario ad hoc para la recogida de los datos sociodemográficos: género, edad, titulación.

- Trait Meta-Mood Scale-24 (TMMS-24; Fernández-Berrocal, Extremera y Ramos, 2004). Este instrumento supone la adaptación española del TMMS-48 elaborado por Salovey et al. (1995). La adaptación española consta de 24 ítems a los que se contesta a través de una escala Likert de 5 puntos $(1=$ Nada de acuerdo; $5=$ Totalmente de acuerdo). Los ítems se distribuyen en tres escalas: Atención Emocional, Comprensión Emocional y Regulación Emocional. La escala está compuesta por tres dimensiones de 8 ítems cada una: Atención a los propios sentimientos, Claridad emocional y Reparación de las emociones. La Atención emocional se define como la capacidad para percibir y expresar los sentimientos de forma adecuada; la Comprensión de sentimientos sería la comprensión de los estados emocionales, y la Reparación emocional, la capacidad de regular los estados emocionales correctamente. El criterio de rangos de IE entre los participantes fue establecido en tres niveles en función de la puntuación mínima (24) y máxima (124). Los valores entre 
92 y 124 indicarían un elevado nivel de IE; entre 58-91, IE moderada; y valores entre 24 y 57, escasa IE.

Se eligió esta prueba por su fácil aplicación, por estar validada para población joven (Salguero, Palomera y Fernández-Berrocal, 2012) y adulta (Fernández-Berrocal et al., 2004). Así, el cuestionario original presenta una validez explicada del $56,4 \%$ de la escala total (el $19,6 \%$ de varianza explicado para el primer factor, el $19,5 \%$ queda explicado por el segundo factor y el 14,07\% para el tercer factor). Asimismo, presenta índices de consistencia interna superiores al ,80. Siguiendo el criterio establecido por Nunnally y Bernstein (1995) se consideraron adecuados índices situados entre 0,70 y 0,80 para el cuestionario total.

Para el presente estudio la fiabilidad (a) fue de 0,83 y la variabilidad analizada a través de la técnica del Análisis Factorial Exploratorio de Componentes Principales (Díaz de Rada Igurquiza, 2002), seguido de rotación Varimax para conseguir una mejor comprensión de la matriz resultante, quedó explicada por el $54,6 \%$.

- Para medir la conducta prosocial de los estudiantes universitarios hacia los compañeros con discapacidad se utilizó la Escala para la Medición de la Conducta Prosocial en Adultos (Prosocialness Scale for Adults [PSA] de Caprara, Capanna, Steca y Paciello, 2005) adaptada específicamente para referirse a la conducta prosocial hacia la discapacidad. Es una escala de dieciséis ítems, tipo Likert con un formato de cinco alternativas de respuesta $(1=$ nunca, $2=$ pocas veces, $3=$ algunas veces, $4=$ a menudo, 5 = habitualmente), en función de la frecuencia con que se den cada una de las conductas descritas. Cada ítem refleja comportamientos y sentimientos que pueden ser incluidos en cuatro factores: de altruismo, empatía, de compartir y de cuidar. El criterio de rangos de conducta prosocial queda establecido en tres niveles en función de la puntuación mínima (20) y máxima (80). Los valores entre 61 y 80 indicarían un elevado nivel de conducta prosocial; entre 41-60, conducta prosocial moderada; y valores entre 20 y 40 , escasa conducta prosocial.

Se ha seleccionado este instrumento por ser breve (requiere aproximadamente 10 minutos su cumplimentación), específico (mide varias dimensiones de prosocialidad) y por sus adecuadas propiedades psicométricas ( $a=, 92$ y varianza explicada del 68\%).

Para la adaptación de este instrumento se siguieron las siguientes fases:

En primer lugar, un lingüista profesional realizó una traducción de la versión original (inglés) a la lengua castellana. Paralelamente a la traducción, el lingüista puntuó en una escala de $0-10$ el grado de dificultad $(0=$ ninguna dificultad; 10 = dificultad máxima $)$ y el grado de equivalencia $(0=$ equivalencia máxima, $10=$ equivalencia mínima). Los ítems se clasificaron según el grado de equivalencia en: 1) ítems sin problemas de adaptación; 2) ítems con problemas en algunas palabras o necesidad de adaptación para su uso en castellano, y 3 ) ítems sin equivalencia cultural. Posteriormente, desde la autoría de investigación y con ayuda del lingüista, se decidió una versión de consenso del cuestionario adaptado al castellano, revisando conjuntamente los ítems y enfocándolos a la conducta prosocial hacia la discapacidad. 
Finalmente, para determinar las propiedades psicométricas del cuestionario en la muestra de este estudio, se calculó la validez a través de la técnica del Análisis Factorial Exploratorio de Componentes Principales (Díaz de Rada Igurquiza, 2002), seguido de rotación Varimax para conseguir una mejor comprensión de la matriz resultante, quedando el $64,07 \%$ de la varianza explicado por la escala (el 25,93\% de varianza explicado para el primer factor, el 15,50\% quedó explicado por el segundo factor, el 14,07\% para el tercer factor y el último factor por el $8,56 \%$ ). Para analizar la fiabilidad del cuestionario se realizó una evaluación de la consistencia interna mediante el coeficiente Alfa de Cronbach, la cual indicó una consistencia adecuada $(a=, 76)$.

\subsection{Procedimiento}

El procedimiento de recogida de información ha consistido en la aplicación del cuestionario a la muestra de alumnos. La muestra ha sido seleccionada intencionalmente entre octubre del 2014 y marzo del 2015, obteniéndose previamente el consentimiento informado de los participantes del estudio. El contacto inicial se realizó directamente proponiéndoles su participación en el estudio. Una vez explicado el proceso se diseñó un formato de los cuestionarios en la Red, creando un apartado específicamente para la línea de investigación a la que se accede a través de un enlace alojado en Google. A continuación, se procedió al proceso de recogida de datos. La recopilación de los cuestionarios se llevó a cabo durante 2 meses.

\subsection{Análisis estadístico}

Para los datos sociodemográficos se obtuvieron las frecuencias y porcentajes. Para identificar los perfiles de IE se utilizó el método de análisis clúster en dos etapas (2-step cluster analysis), es una herramienta exploratoria diseñada para revelar las agrupaciones naturales dentro de un conjunto de información que, de otra manera, no sería evidente. Asimismo, el procedimiento puede determinar automáticamente el número óptimo de clústeres.

Los perfiles se han definido a partir de las diferentes combinaciones de las tres dimensiones de IE que evalúa la Escala TMMS-24 de Fernández-Berrocal et al. (2004): Atención, Comprensión y Regulación Emocional. Posteriormente, se realizaron análisis de varianza en los clústeres obtenidos con el objetivo de analizar la significación estadística de las diferencias existentes entre los grupos en los factores de conducta prosocial. Finalmente, se realizaron pruebas post hoc para identificar entre qué grupos se encontraban las diferencias. Se utilizó el método de Scheffé al no estar cada grupo compuesto por el mismo número de participantes. Para analizar la magnitud o tamaño del efecto de estas diferencias, se atendió al índice directo de eta ${ }^{2}$. Asimismo, se calculó el tamaño del efecto (diferencia media tipificada o índice d; Cohen, 1988) para calcular la magnitud de las diferencias observadas. Los datos se analizaron por medio del paquete estadístico SPSS versión 19.0. 


\section{Resultados}

\subsection{Identificación de perfiles de IE}

El método clúster, tratando de lograr la máxima homogeneidad en cada grupo y las mayores diferencias entre ellos, determinó 3 agrupaciones de las dimensiones de IE. De esta forma, quedó integrada por un grupo formado por 104 (31,80\%) estudiantes con altas habilidades en las tres dimensiones de IE (clúster 1), un grupo con predominio de habilidad de Comprensión y Regulación (clúster 2) formado por 123 estudiantes $(37,61 \%)$ y un tercer grupo con predominio de bajas habilidades en IE (clúster 3) conformado por 100 (30,58\%) estudiantes (véase Figura 1).

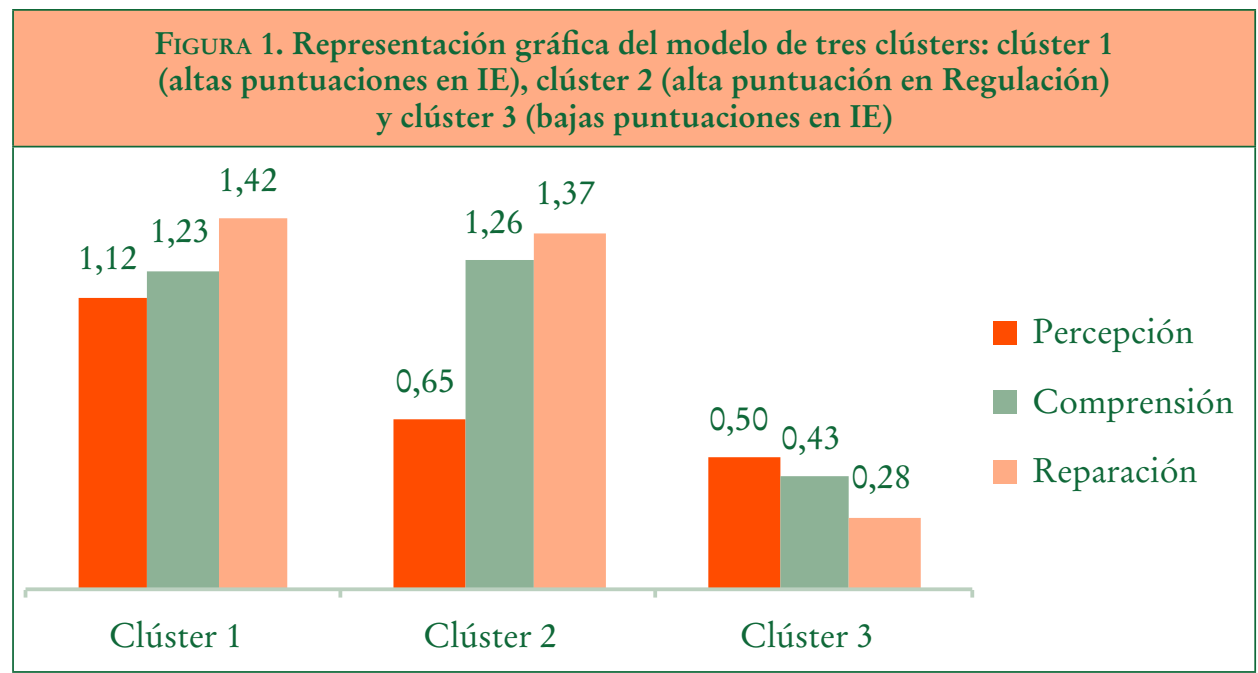

\subsection{Diferencias intergrupo en dimensiones de conducta prosocial}

$\mathrm{Al}$ atender a las puntuaciones medias de la escala global se observaron diferencias estadísticamente significativas en los tres clústers $\left(F_{(2,324)}=5,88, p<, 05, \eta^{2}=, 33\right)$, encontrándose que el grupo 1 indicó medias superiores al grupo $3(d=0,52)$. Asimismo, el grupo 2 mostró puntuaciones medias más elevadas que el grupo $3(d=0,44)$.

Al examinar los factores y las comparaciones post hoc para ver entre qué grupos se encontraban las diferencias se observaron, con respecto al factor 1: Altruismo, que el grupo de altas puntuaciones en las tres dimensiones de IE, es decir, el grupo 1 y el Grupo 2, indicaron tener medias significativamente más elevadas que el grupo 3, $\left(F_{(2,324)}=4,45, p<, 05, \eta^{2}=, 44\right)$, siendo el tamaño del efecto moderado en el análisis post hoc entre el grupo 3 y el grupo $2(d=0,20)$ y elevado entre la comparación del grupo 3 con el grupo $1(d=0,44)$. 
En lo relativo al factor 2, relativo a la Empatía, se observó que el grupo $1(d=0,42)$ y el grupo $2(d=0,24)\left(F_{(2,324)}=3,21, p<, 05, \eta^{2}=, 38\right)$ indicaron medias superiores al grupo 3.

La misma dirección se encontró en el factor 3, relativo a Compartir y en el factor 4, concerniente al Apoyo. En este caso, en el factor relativo a Compartir (factor 3), se observó que el grupo $1(d=0,35)$ y el grupo $2(d=0,32)$ indicaron puntuaciones más elevadas que el grupo $3\left(F_{(2,324)}=6,09, p<, 05, \eta^{2}=, 28\right)$. En lo concerniente al factor 4, Apoyo, también se observaron diferencias estadísticamente significativas entre los tres clústers, destacando el grupo 1, con puntuaciones medias superiores al grupo $3\left(F_{(2,324)}=1,27, p<, 001, \eta^{2}=, 42, d=0,41\right)$, y puntuaciones medias al grupo $2(d=0,38)$.

\begin{tabular}{|c|c|c|c|c|c|c|c|c|c|c|c|}
\hline $\begin{array}{l}\text { TABLA } 2 . \mathrm{Me} \\
\text { de eta cuad }\end{array}$ & $\begin{array}{l}\text { s y d } \\
0\left(n^{2}\right.\end{array}$ & Va & $\begin{array}{l}\text { ones t } \\
\text { cada t }\end{array}$ & . & te & 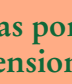 & los $t_{1}$ & \% & $s$ y & & \\
\hline \multirow[t]{2}{*}{$\begin{array}{l}\text { Factores de escala } \\
\text { conducta prosocial }\end{array}$} & \multicolumn{2}{|c|}{$\begin{array}{l}\text { Grupo } 1 \\
\text { Alta IE }\end{array}$} & \multicolumn{2}{|c|}{$\begin{array}{c}\text { Grupo } 2 \\
\text { Alta Regul. } \\
\text { Alta Comp. } \\
\text { Baja Aten. }\end{array}$} & \multicolumn{2}{|c|}{$\begin{array}{l}\text { Grupo } 3 \\
\text { Baja IE }\end{array}$} & \multicolumn{2}{|c|}{ Total } & \multirow[t]{2}{*}{$\begin{array}{c}F \\
(2,324)\end{array}$} & \multirow[t]{2}{*}{$p$} & \multirow[t]{2}{*}{$\eta^{2}$} \\
\hline & $M$ & $D T$ & $M$ & $D T$ & $M$ & $D T$ & $M$ & $D T$ & & & \\
\hline Factor 1 Altruismo & 12.56 & 2,22 & 11,74 & 2,44 & 11,27 & 2,88 & 11,75 & 2,57 & 4,458 & 012 & ,46 \\
\hline Factor 4 Empatía & 10,47 & 2,36 & 10,31 & 2,69 & 9,59 & 2,07 & 10,13 & 2,49 & 3,217 & ,007 & ,38 \\
\hline Factor 2 Compartir & 7,73 & 2,56 & 7,38 & 2,34 & 6,50 & 2,19 & 7,19 & 2,37 & 6,090 & ,003 & ,182 \\
\hline Factor 3 Aроуо & 7,89 & 1,62 & 6,81 & 1,95 & 6,46 & 1,99 & 6,72 & 1,91 & 1,273 &, 000 & ,42 \\
\hline Total & 40,04 & 6,91 & 38,46 & 7,47 & 36,00 & 7,30 & 38,03 & 7,44 & 5,883 & ,003 & , 185 \\
\hline
\end{tabular}

\subsection{Diferencias intergrupo en conducta prosocial hacia diferentes tipos de discapacidad}

Finalmente, con respecto a las comparaciones entre grupos en conducta prosocial de los estudiantes en función de los diferentes tipos de discapacidades se observaron diferencias estadísticamente significativas en función de esta variable, encontrándose que las puntuaciones medias de conducta prosocial indicaron ser más elevadas hacia la discapacidad motora y hacia la discapacidad visual al compararlas con la discapacidad auditiva y la intelectual.

TABLA 3. Puntuaciones medias en conducta prosocial hacia diferentes tipos de discapacidad

\begin{tabular}{|c|c|c|c|c|c|}
\hline & $N$ & $M$ & D.T. & $\underset{(3.323)}{F}$ & $p$ \\
\hline Física & 102 & 38,99 & 7,10 & \multirow{4}{*}{2,18} & \multirow{4}{*}{0,46} \\
\hline Intelectual & 69 & 36,17 & 6,99 & & \\
\hline Visual & 83 & 38,96 & 7,95 & & \\
\hline Auditiva & 73 & 37,09 & 7,54 & & \\
\hline Total & 327 & 38,03 & 7,44 & & \\
\hline
\end{tabular}




\section{Discusión}

Este trabajo tuvo varios objetivos. El primero de ellos fue analizar las distintas combinaciones de las dimensiones de IE en una muestra de estudiantes universitarios para identificar diferentes perfiles de razonamiento emocional. Una vez identificados estos perfiles, el segundo objetivo consistió en comprobar si existían diferencias estadísticamente significativas entre los grupos obtenidos en función de las dimensiones de la conducta prosocial, dirigida concretamente al comportamiento prosocial hacia los estudiantes con discapacidad. La idea de la que parte este estudio es que si la IE guarda relación con la conducta prosocial y ambos constructos son dinámicos, profundizar sobre dicha relación puede ayudar a comprender el comportamiento y las creencias de los estudiantes hacia los compañeros con discapacidad y, con ello, diseñar programas de intervención para el fomento de estos constructos para mejorar la integración de estudiantes con discapacidad.

Los resultados del primer objetivo, identificar si entre los participantes existen combinaciones de diferentes dimensiones de IE que den lugar a diferentes perfiles, mostraron la existencia de perfiles con diferentes combinaciones en las dimensiones de IE. Así, mediante el análisis de conglomerados se identificaron tres perfiles distintos, confirmándose la primera de las hipótesis. Un grupo con un perfil de altas puntuaciones en los tres componentes de IE (grupo 1), un segundo grupo con puntuaciones bajas en Atención y altas en Comprensión y Regulación emocional (grupo 2) y, por último, un grupo con puntuaciones bajas en las tres dimensiones de IE (grupo 3). Estos perfiles permitirían justificar los resultados obtenidos en investigaciones previas. Así, aquellos estudios en los que se ha vinculado positivamente la alta IE con variables de tipo académico, personal y social (Martins et al., 2010; Pena y Repetto, 2008) podrían estar vinculados con el perfil de altas puntuaciones en las tres dimensiones (grupo 1). Asimismo, los trabajos que destacan la relación diferencial existente entre las dimensiones de la IE y diferentes variables relacionadas con el ajuste interpersonal y social (p. e., habilidades sociales) (Berbena, Sierra y Vivero, 2008; Fernández Berrocal et al., 2004) respaldarían el perfil en el que destacan puntuaciones altas en comprensión y regulación y bajas en atención (grupo 2). Finalmente, bajas puntuaciones en las tres dimensiones de IE estarían relacionadas con déficits en el bienestar y ajuste psicológico, menor cantidad y calidad de las relaciones interpersonales, así como una mayor aparición de conductas disruptivas (Extremera y Fernández-Berrocal, 2003; Salguero et al., 2012), esto apuntaría la existencia de un perfil de baja IE (grupo 3).

Con respecto al segundo objetivo, los resultados dan respaldo a la segunda hipótesis planteada, es decir, las puntuaciones medias de los clústeres hallados revelaron diferencias estadísticamente significativas en la mayoría de factores de conducta prosocial. Estos datos ofrecen consistencia a la validez de la existencia de diferentes perfiles de IE y ayudan a profundizar en la relación entre IE y conducta prosocial.

Al observar las puntuaciones medias de cada factor o dimensión de la conducta prosocial, se refleja que resaltan especialmente los grupos en los que las dimensiones de IE relativas a la Comprensión y a la Regulación emocional son elevadas, esto es, en el grupo 1 y en el grupo 2. Esta idea queda reforzada al examinar el tamaño del efecto, 
el cual indica que, en la mayoría de factores de la conducta prosocial (p. e. ayudar, compartir y ser empático), la magnitud de estas diferencias es elevada con respecto al grupo de bajas puntuaciones en estas dimensiones (grupo 3). Por ejemplo, si se atiende al factor 1, referente a la ayuda o altruismo, se observa que el grupo con elevada IE (grupo 1) y el grupo de alta Comprensión y Regulación emocional (grupo 2) destacan con puntuaciones medias superiores al resto de grupos.

Estos resultados están en línea con los obtenidos por Carreres-Ponsoda, EscartíCarbonell, Cortell-Tormo, Fuster-Lloret y Andreu-Cabrera (2012), al analizar el altruismo y la IE, en los cuales se observó una relación significativa entre ambos constructos. En este sentido, el altruismo hace referencia a las conductas que benefician a otros, provocando o manteniendo efectos positivos, y que quien las lleva a cabo lo hace voluntariamente, con la intención de ayudar a otros y sin anticipar recompensas externas a corto o a largo plazo (Morales, Fernández, Infante, Trianes y Cerezo, 2010). De este modo, el componente de Comprensión ayudaría a entender a los demás y el siguiente, Regulación emocional, ayudaría a expresar las emociones adecuadas.

Los resultados encontrados refuerzan los obtenidos al examinar el siguiente factor de conducta prosocial, la empatía, observándose que los grupos que presentan puntuaciones elevadas en las dimensiones de Regulación y Comprensión emocional (grupo 1 y grupo 2) reflejan más empatía que el grupo con puntuaciones bajas en estas dimensiones de IE.

Como en líneas anteriores se ha citado, la empatía puede definirse como «una respuesta afectiva de comprensión sobre el estado emocional de otros, que induce a sentir el estado en que se encuentra el otro» (Eisenberg et al., 2005) y, por tanto, puede considerarse como el motivador fundamental de la conducta altruista y como la habilidad que las origina (Eisenberg et al., 2005). Esta habilidad permitiría a las personas enlazar con las sutiles señales sociales del otro indicando qué necesitan de los demás.

En este sentido, la literatura sobre la temática de la IE vincula otros factores característicos de la conducta prosocial, como las dimensiones de compartir y apoyo o cuidado (Betina Lacunza y Contini de González, 2011). Esta asociación queda reflejada en los resultados del presente estudio, observándose que los estudiantes que presentaron puntuaciones más altas en los factores de compartir y apoyo y cuidado conforman los perfiles de IE en los que destaca el componente de Comprensión y Regulación emocional (grupos 1 y 2).

Estos datos son congruentes con los presentados por Extremera y Fernández Berrocal (2003). Estos autores, basándose en la hipótesis de que unos niveles adecuados de comprensión y regulación de las emociones son necesarios para unas relaciones sociales positivas, examinaron las conexiones entre IE y la calidad de las relaciones interpersonales en una muestra de jóvenes, observaron que los participantes con puntuaciones más elevadas en Comprensión y Regulación emocional mostraron también puntuaciones más altas en los factores positivos de apoyo social (compañerismo, intimidad, afecto y alianza) y puntuaciones más bajas en los factores de interacción negativa (conflicto e inhibición emocional).

Sobre la relación encontrada entre estos factores, los resultados están en concordancia con las otras dimensiones de conducta prosocial, esto es, altruismo y empatía. 
Así, cuando una persona observa que otra necesita su apoyo, es ahí cuando pueden surgir emociones que den lugar a que la persona sea empática, se motive a realizar acciones altruistas y comparta y ayude al otro, constituyendo un punto de enlace entre ambas variables, es decir, entre IE y conducta prosocial.

Con respecto al tercer objetivo, explorar si la conducta prosocial de los estudiantes hacia las personas con discapacidad puede estar influida según la tipología de la discapacidad, en los resultados se observa que los estudiantes tienen más preferencia en dirigir estos comportamientos de ayuda hacia personas con discapacidad motora y visual que a personas con discapacidad auditiva e intelectual. Es posible que esto pueda explicarse porque la visibilidad de la discapacidad facilite los comportamientos prosociales. Así, posiblemente, la sociedad sea más proclive a ayudar a personas con discapacidad motora y visual, pues son discapacidades visibles y, por tanto, reflejen en mayor medida más necesidad de apoyo que las personas con discapacidades invisibles como la discapacidad auditiva e intelectual (Suriá, 2018).

A la luz de los resultados obtenidos, se deduce que la IE está fuertemente asociada a otras características que conforman la personalidad como es la conducta prosocial, lo que va a favorecer unas competencias sociales más altruistas y, por tanto, mayor comprensión, tolerancia y apoyo por parte de los estudiantes hacia sus compañeros con discapacidad. Por otro lado, estudiantes con baja IE tienen tendencia a ser menos tolerantes con la discapacidad y, por tanto, ser población con la que haya que intervenir con objeto de mejorar sus creencias y competencias sociales.

Sin embargo, no hay que pasar por alto algunas limitaciones de este trabajo. La principal dificultad reside en la conceptualización de la IE y su forma de medida. En la actualidad existen múltiples cuestionarios que tratan de analizar la IE, lo cual dificulta la comparación de resultados entre diferentes estudios. Del mismo modo, habría sido pertinente averiguar no únicamente la conducta prosocial, sino también las actitudes de los estudiantes hacia la discapacidad y comprobar su relación.

Con respecto a la muestra de estudio, los datos se refieren a población universitaria, con lo que no serían extrapolables a estudiantes de otras edades o culturas.

De la misma forma, los participantes de este estudio se ofrecieron voluntariamente para cumplimentar los cuestionarios, por lo que estos podrían tener diferentes expectativas y motivaciones para participar en comparación con las personas que se mostraron reacias a participar. En futuras investigaciones se debería controlar este factor con el objeto de incrementar la validez interna de los resultados.

A pesar de estas limitaciones se considera que los resultados de este trabajo resultan interesantes, pues destacan que, si bien la IE se relaciona con un mejor ajuste de los individuos, no todas las dimensiones de la IE contribuyen de igual forma a este ajuste. De hecho, tal y como se pone de manifiesto en este estudio, los estudiantes que presentan un patrón caracterizado por alta regulación y baja atención tienden a presentar puntuaciones en la conducta prosocial más altas que los estudiantes con una puntuación alta en atención y baja en regulación.

Así, se considera que este trabajo contribuye al conocimiento de la IE y la conducta prosocial de los estudiantes hacia los compañeros con discapacidad, lo que podría resultar relevante para el diseño de programas de intervención para mejorar la 


\section{CONDUCTA PROSOCIAL DE LOS ESTUDIANTES HACIA LA DISCAPACIDAD \\ Y RELACIÓN CON LA INTELIGENCIA EMOCIONAL \\ RAQUEL SURIÁ MARTÍNEZ}

integración de estudiantes con discapacidad. De este modo, el abordaje del estudio de los comportamientos prosociales desde el área educativa puede ayudar no solo a detectar estas conductas, sino a crear proyectos y medios para que los estudiantes cambien sus actitudes y refuercen estas conductas prosociales.

\section{Referencias bibliográficas}

Ainscow, M. (2005). Para comprender el desarrollo del Sistema Educativo Inclusivo. Electronic Journal of Research in Educational Psychology, 3, 5-20.

Arnáiz-SÁnchez, P. (2012). Escuelas eficaces e inclusivas: cómo favorecer su desarrollo. Educatio Siglo XXI, 30 (1), 25-44.

Bandura, A., Caprara, G. V., Barbaranelli, C., Gerbino, M. y Pastorelli, C. (2003). Role of affective self-regulatory efficacy in diverse spheres of psychosocial functioning. Child development, 74 (3), 769-782.

Berbena, M. A., Sierra, M. D. y Vivero, M. D. (2008). Inteligencia emocional y habilidades sociales en adolescentes con alta aceptación social. Electronic Journal of Research in Educational Psychology, 6 (15), 321-338.

Betina Lacunza, A. y Contini de González, N. (2011). Las habilidades sociales en niños y adolescentes. Su importancia en la prevención de trastornos psicopatológicos. Fundamentos en Humanidades, 12 (23).

Brackett, M. A., Rivers, S. E., Shiffman, S., Lerner, N. y Salovey, P. (2006). Relating emotional abilities to social functioning: a comparison of self-report and performance measures of emotional intelligence. Journal of Personality and Social Psychology, 91, 780-795.

Brown, E. y Ferris, J. M. (2007). Social Capital and Philanthropy: An Analysis of the Impact of Social Capital on Individual Giving and Volunteering. Nonprofit and Voluntary Sector Quarterly, 36 (1), 85-99.

Buenrostro, A., Valadez, D., Soltero, R., Nava, G., Zambrano, R. y García, A. (2012). Inteligencia emocional y rendimiento académico en adolescentes. Revista de Educación y Desarrollo, 20, 29-37.

Caprara, G. V., Alessandri, G. y Eisenberg, N. (2012). Prosociality: The contribution of traits, values, and self efficacy beliefs. Journal of Personality and Social Psychology, 102 (6), 1289-1303.

Caprara, G. V., Capanna, C., Steca, P. y Paciello, M. (2005). Misura e determinanti personali della prosocialità. Un approccio sociale cognitivo. Giornale Italiano di Psicologia, 32 (2), 287-308.

Caprara, G. V. y Pastorelli, C. (1993). Early emotional instability, prosocial behavior, and aggression: some methodological aspects. European Journal of Personality, 7 (1), 19-36.

Carlo, G., Crockett, L. J., Randall, B. A. y Roesch, S. C. (2007). A latent growth curve analysis of prosocial behavior among rural adolescents. Journal of Research on Adolescence, 17 (2), 301-324.

Carreres-Ponsoda, F., Escartí-Carbonell, A., Cortell-Tormo, J. M., Fuster-Lloret, V. y ANdreu-Cabrera, E. (2012). The relationship between out-of-school sport participation and positive youth development. Journal of Human Sport and Exercise, 7, 671-683.

Cohen, J. (1988). Statistical Power Analysis for the Behavioral Sciences. New York, NY: Academic Press. 


\section{CONDUCTA PROSOCIAL DE LOS ESTUDIANTES HACIA LA DISCAPACIDAD \\ Y RELACIÓN CON LA INTELIGENCIA EMOCIONAL RAQUEL SURIÁ MARTÍNEZ}

Damm-MuÑoz, X. (2014). Representaciones y actitudes del profesorado frente a la integración de niños/as con necesidades educativas especiales al aula común. Revista Latinoamericana de Educación Inclusiva, 3, 25-35.

Del Barco, B. L., Castaño, E. F. y Carroza, T. G. (2010). Variables individuales que influyen en las actitudes hacia la inmigración en el ámbito escolar. Anales de Psicología, 26 (2), 359-368.

De Boer, A. A., Pijl, S. J. y Minnaert, A. E. M. G. (2010). Attitudes of parents towards inclusive education: A review of the literature. European Journal of Special Needs Education, $25,165-181$.

Díaz de Rada Igurquiza, V. (2002). Análisis Factorial. En V. Díaz de Rada Igurquiza, Técnicas de análisis multivariante para investigación social y comercial (pp. 91-156). Madrid: RA-MA.

Echeita, G. y Simón, C. (2014). La contribución de la educación escolar a la calidad de vida de las personas con discapacidad. Ante el desafío de su inclusión social. En De Lorenzo, R. y Pérez Bueno, L. C. (Coords.), Tratado sobre Discapacidad. Madrid: Thomson \& Aranzadi.

Eisenberg, N., Zhou, Q., Spinrad, T. L., Valiente, C., Fabes, R. y Liew, J. (2005). Relations among positive parenting, children's effortful control, and externalizing problems: A threewave longitudinal study. Child Development, 76, 055-1071.

Esteban Guitart, M., Rivas-Damián, M. J. y Pérez Daniel, M. R. (2012). Empatía y tolerancia a la diversidad en un contexto educativo intercultural. Universitaria. Psycholy, 11 (2), 415-426. Bogotá, Colombia.

Extremera, N. y Fernández-Berrocal, P. (2003). La inteligencia emocional en el contexto educativo: hallazgos científicos de sus efectos en el aula. Revista de Educación, 332, 97-116.

Fehr, E. y Fischbacher, U. (2003). The Nature of Human Altruism. Nature, 425, 785-791.

Fernández-Berrocal, P., Extremera, N. y Ramos, N. (2004). Validity and reliability of the Spanish modified version of the Trait Meta-Mood Scale. Psychological Reports, 94, 751-755.

Gilar, R., Miñano, P. y Castejón, J. L. (2008). Inteligencia emocional y empatía: su influencia en la competencia social en Educación Secundaria Obligatoria. SUMMA Psicológica UST, 5 (1), 21-32.

Hardy, S. A., Carlo, G. y Roesch, S. C. (2010). Links Between Adolescents Expected Parental Reactions and Prosocial Behavioral Tendencies: The Mediating Role of Prosocial Values. Journal Youth Adolescence, 39, 84-95.

Inglés, C. J., Martínez-González, A. E. y García-Fernández, J. M. (2015). Conducta prosocial y estrategias de aprendizaje en una muestra de estudiantes españoles de Educación Secundaria Obligatoria. European Journal of Education and Psychology, 6 (1).

Jiménez, M. I. y López-Zafra, E. (2011). Actitudes sociales y adaptación social en adolescentes españoles: el papel de la inteligencia emocional percibida. Revista de Psicología Social, 26 (1), 105-117.

Lazarus, R. (2000). Estrés y emoción. Manejo e implicaciones en nuestra salud. España, Bilbao: Desclée de Brouwer.

Lim, K. M., Khoo, A. y Wong, M. Y. (2007). Relationship of Delinquent Behaviors to Prosocial Orientations of Adolescents. North American Journal of Psychology, 9, 1, 183-188.

Lozano, A. M. y Etxebarria, I. (2007). La tolerancia a la diversidad en los adolescentes y su relación con la autoestima, la empatía y el concepto del ser humano. Infancia y Aprendizaje, 30 (1), 109-129. 
Martins, A., Ramalho, N. y Morin, E. (2010). A comprehensive meta-analysis of the relationship between emotional intelligence and health. Personality and Individual Differences, 49, 554-564.

Martorell, M. C., González, R., Aloy, M. y Ferris, M. C. (1995). Socialización y conducta prosocial. Revista Iberoamericana de Diagnóstico y Evaluación Psicológica, 1, 73-102.

Mayer, J. D. y Salovey, P. (1997). What is emotional intelligence? En P. Salovey y D. SLUYTER (Eds.), Emotional development and emotional intelligence: Educational Implications (pp. 3-31). New York: Perseus Books Group.

Morales, F. M., Fernández, F. J., Infante, L., Trianes, M. V. y Cerezo, M. T. (2010). Eficacia de una intervención para incrementar apoyo social en adolescentes discapacitados motores a partir del voluntariado de estudiantes de educación secundaria. Revista Latinoamericana de Psicología, 41 (1), 141-150.

Morales, M. I. J. y Zafra, E. L. (2013). Impacto de la Inteligencia Emocional percibida, actitudes sociales y expectativas del profesor en el rendimiento académico. Electronic Journal of Research in Educational Psychology, 11 (29), 75-98.

Novo-Corti, I., Muñoz-Cantero, J. y Calvo-Porral, C. (2011). Análisis de las actitudes de los jóvenes universitarios hacia la discapacidad: un enfoque desde la teoría de la acción razonada. Revista Electrónica de Investigación Educativa, 17, 1-26.

Nunnally, J. C. y Bernstein, I. J. (1995). Teoría psicométrica. Madrid: McGraw-Hill.

Olivar, R. R. y Soza, P. E. (2014). Comunicación prosocial en familias e hijos con discapacidad. Italian Journal of Special Education for Inclusion, 2 (1), 13-21.

Parra, D. J. L. y Luque-Rojas, M. J. (2011). Conocimiento de la discapacidad y relaciones sociales en el aula inclusiva. Sugerencias para la acción tutorial. Revista Iberoamericana de Educación, 54 (6), 3.

Pena, M. y Repetto, E. (2008). Estado de la investigación en España sobre inteligencia emocional (IE) en el ámbito educativo. Electronic Journal of Research in Educational Psychology, 15, 400-420.

Redondo-Pacheco, J. y Guevara-Melo, E. (2012). Diferencias de género en la prevalencia de la conducta prosocial y agresiva en adolescentes de dos colegios de la ciudad de PastoColombia. Revista Virtual Universidad Católica del Norte, 1 (36), 173-192.

Salguero, J. M., Palomera, R. y Fernández-Berrocal, A. (2012). Perceived emotional intelligence as predictor of psychological adjustment in adolescents: a 1-year prospective study. European Journal of Psychology of Education, 27, 21-34.

Salovey, P., Mayer, J. D., Goldman, S., Turvey, C. y Palfai, T. (1995). Emotional attention, clarity, and repair: Exploring emotional intelligence using the Trait Mood Scale. En J. W. Pennebaker (Ed.), Emotion, disclosure and health (pp. 125-154). Washington DC: American Psychological Association.

Spinrad, T. L., Eisenberg, N., Cumberland, A., Fabes, R. A., Valiente, C., Shepard, S. A. ... y Guthrie, I. K. (2006). Relation of emotion-related regulation to Children's social competence: A longitudinal study. Emotion, 6 (3), 498-510.

Suriá, R. (2014). Actitudes integradoras en el contexto educativo de los estudiantes con discapacidad según la percepción de sus progenitores. Bordón. Revista de Pedagogía, 66, 157-172.

SURIÁ, R. (2016). Relationships between self-concept and resilience profiles in young people with disabilities. Electronic Journal of Research in Educational Psychology, 3, 450-473.

SURIÁ, R. (2018). Inteligencia emocional y bienestar subjetivo en estudiantes con discapacidad sobrevenida. Bordón, 1, 125-140. 


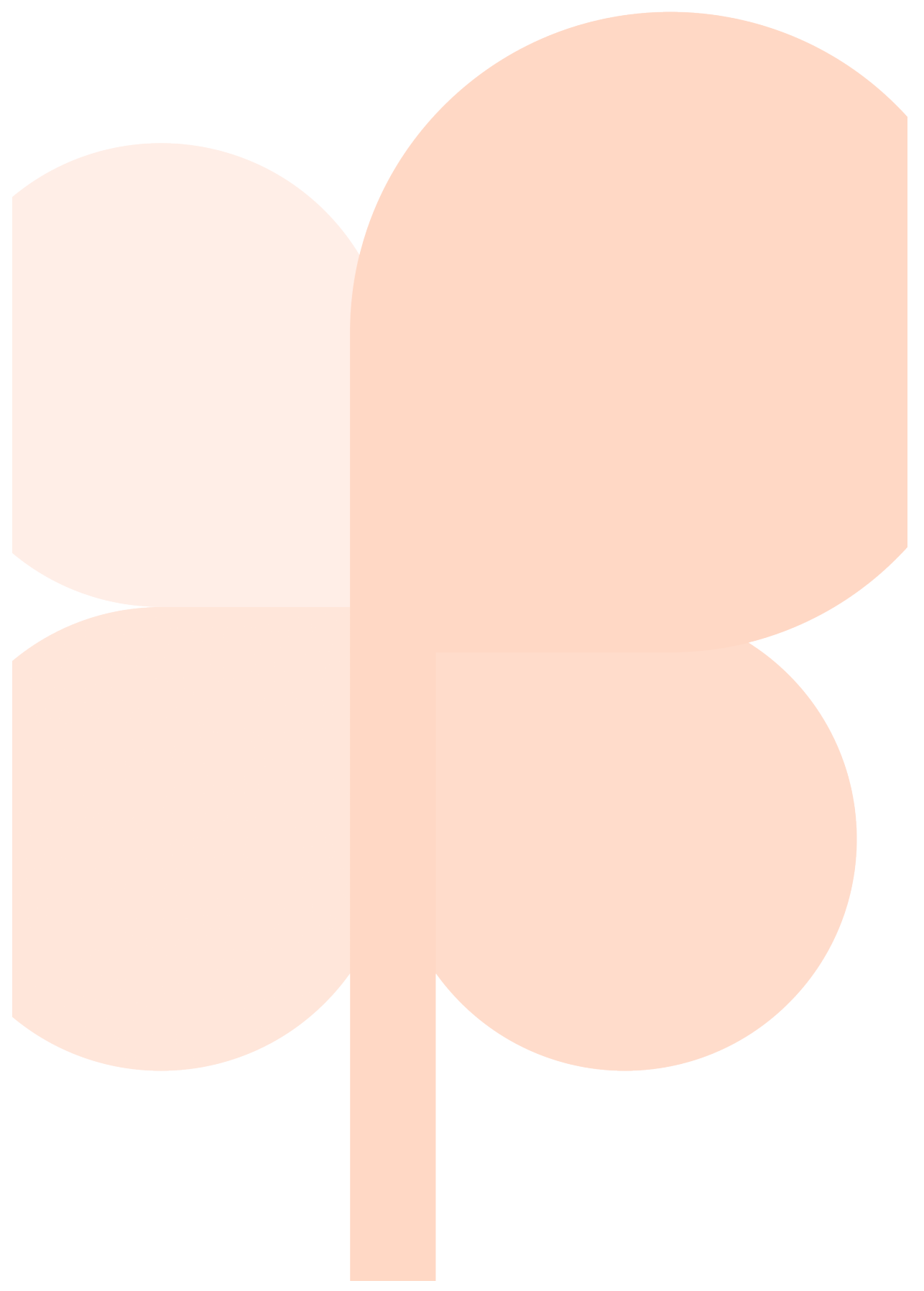

